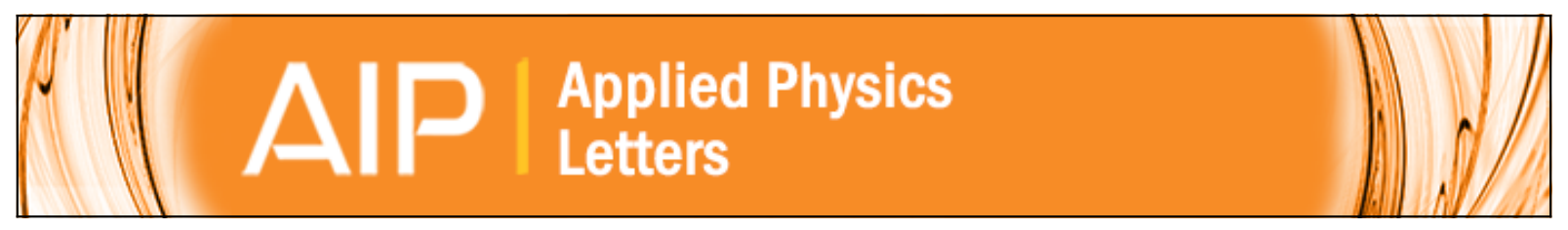

\title{
Extremely low frequency response of magnetoelectric multilayer composites
}

Shuxiang Dong, Junyi Zhai, Zhengping Xing, Jie-Fang Li, and D. Viehland

Citation: Applied Physics Letters 86, 102901 (2005); doi: 10.1063/1.1881784

View online: http://dx.doi.org/10.1063/1.1881784

View Table of Contents: http://scitation.aip.org/content/aip/journal/apl/86/10?ver=pdfcov

Published by the AIP Publishing

\section{Over 700 papers \&}

presentations on

multiphysics simulation visw now

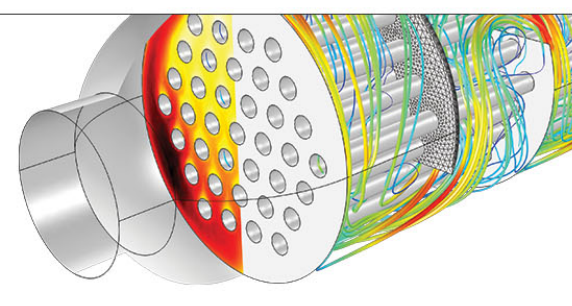




\title{
Extremely low frequency response of magnetoelectric multilayer composites
}

\author{
Shuxiang Dong, ${ }^{\text {a) }}$ Junyi Zhai, Zhengping Xing, Jie-Fang Li, and D. Viehland \\ Materials Science \& Engineering, Virginia Tech, Blacksburg, Virginia 24061
}

(Received 11 October 2004; accepted 1 February 2005; published online 28 February 2005)

A promising generation of extremely low frequency magnetic field sensors, based on multilayer composites (MLCs) of magnetostrictive Terfenol-D $\left(\mathrm{Tb}_{1-x} \mathrm{Dy}_{x} \mathrm{Fe}_{2-y}\right)$ and piezoelectric $\mathrm{Pb}\left(\mathrm{Mg}_{1 / 3} \mathrm{Nb}_{2 / 3}\right) \mathrm{O}_{3}-\mathrm{PbTiO}_{3}$, has been developed. Our MLC magnetoelectric sensor presently shows a limit in (i) working frequency of $\sim 5 \times 10^{-3} \mathrm{~Hz}$; and (ii) magnetic field sensitivity of $10^{-7}, 10^{-9}$, and $10^{-11} \mathrm{~T}$ for frequencies of $f=10^{-2}, 1$, and $10^{2} \mathrm{~Hz}$, respectively. The results open up possibilities for sensitive low frequency passive magnetic anomaly detection. () 2005 American Institute of Physics. [DOI: 10.1063/1.1881784]

The magnetoelectric (ME) effect ${ }^{1}$ in materials which are simultaneously ferromagnetic and ferroelectric has been a research topic in recent years, offering potential for magnetic field sensors. ME materials of single phase, multiple phases, and laminate composites have been reported. ${ }^{2-12}$ The magnetostrictive-piezoelectric layered laminates have been shown to have much better ME properties than the others. Recently, laminate composite designs have been developed that have higher induced ME voltages. ${ }^{10-12}$ These laminates consist of longitudinally magnetized and transversely polarized (or L-T) layers of magnetostrictive Terfenol-D and piezoelectric $\mathrm{Pb}(\mathrm{Zr}, \mathrm{Ti}) \mathrm{O}_{3}$.

An important consideration in the design and operation of any magnetic field sensor is the frequency bandwidth of detectable magnetic fields. Different applications require sensitivity over different bandwidths. For example, magnetic anomaly detectors $^{13}$ require sensitivity to frequencies as low as $10^{-3} \mathrm{~Hz}$, whereas magnetocardiography ${ }^{14}$ and magnetoencephalography ${ }^{14,15}$ require high sensitivity between $10^{-2}$ and $10^{3} \mathrm{~Hz}$.

Unfortunately, prior studies by Podney ${ }^{16}$ of ME laminates of two or three layers have shown a roll-off of the induced $M E$ voltage with decreasing frequency, beginning near a low frequency cutoff limit of $f_{\text {cut }} \approx 10 \mathrm{~Hz}$. At this frequency limit, the amplitude of the detected signal was reduced by a factor of $1 / \sqrt{2}$. However, in this letter, we will show that multilayer composites (MLCs) can detect magnetic field variations of milli-Hz frequency.

To lower the limit of the working frequency and to enhance the weak signal response, we have developed (L-T) mode ME-MLCs. Figure 1 illustrates the 11-layer laminate design that we focused on in this investigation. It consists of five (001)-oriented $\mathrm{Pb}\left(\mathrm{Mg}_{1 / 3} \mathrm{Nb}_{2 / 3}\right) \mathrm{O}_{3}-30 \% \mathrm{PbTiO}_{3}$ (or PMN-PT) layers, each sandwiched between two Terfenol-D $\left(\mathrm{Tb}_{1-x} \mathrm{Dy}_{x} \mathrm{Fe}_{2-y},\right)$ ones. It uses the same L-T configuration as our prior three-layer design, ${ }^{11,12}$ where the polarization of each successive piezoelectric layer is reversed with respect to the prior, along the thickness direction. The length and width of each layer is 12.7 and $6 \mathrm{~mm}$, respectively; and the total laminate cross-sectional area $A$ is $6 \times 8.5 \mathrm{~mm}^{2}$. Theoretically from a mechanics perspective, by keeping the thickness ratio

a) Author to whom correspondence should be addressed; electronic mail:
sdong@mse.vt.edu of all $N$ piezoelectric layers and all $N+1$ magnetostrictive ones constant, the ME voltage coefficient should be independent of the total number of layers. It is because all $N$ piezoelectric layers are connected electronically in parallel. However, in applications as a sensor, a multilayer configuration will increase the output signal, because the induced charge from the ME-MLC will be $N$ times that from a laminate containing only one piezoelectric layer.

Since the layers are electrically connected in parallel and the Terfenol-D ones can be considered as good conductors, the total capacitance of the MLC is also increased by $N$ times (i.e., $N C_{0}$, where $C_{0}$ is the capacitance of an individual piezoelectric layer and $N$ is the number of piezoelectric layers). Accordingly, we have approached lowering $f_{\text {cut }}$ by a multilayer design that increases $C_{\mathrm{o}}$. Assuming that the magnetic field to be sensed is sinusoidal, the cutoff frequency for detection is $f_{\text {cut }}=\frac{1}{2 \pi \tau}$, where $\tau=N R C_{0}$ is the time constant, and $R$ is the less of either the laminate's resistance or the input resistance of an electrometer. As a result, the value of $f_{\text {cut }}$ is decreased by a factor of $1 / N$ by increasing the piezoelectric layer number to $N$. In addition, an electrometer with high input resistance $\left(>10^{9} \Omega\right)$ is important to obtain a large time constant $\tau$.

Measurements of the induced ME voltage were performed in a magnetically shielded environment made of $\mu$ metal, which had a noise rejection capability of $\sim 10^{5}$. Our low frequency measurement system used an op-amp with high input resistance to collect the charge from the sample

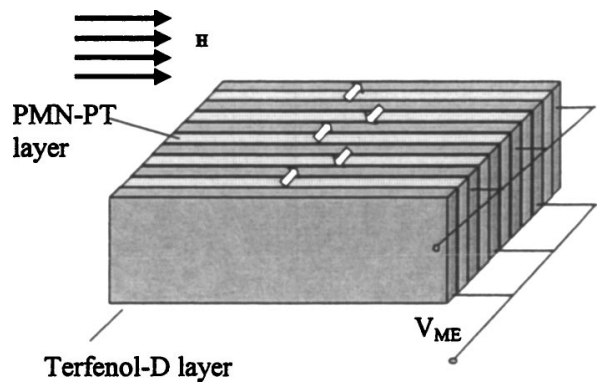

FIG. 1. Our long-type configuration of a L-T mode magnetoelectric multilayer composite (i.e., ME-MLC). The laminate design consists of five PMN-PT layers, each sandwiched between two Terfeonl-D ones. It uses the same L-T mode configuration as previously reported for our prior threelayer design (see Refs. 11,12). 


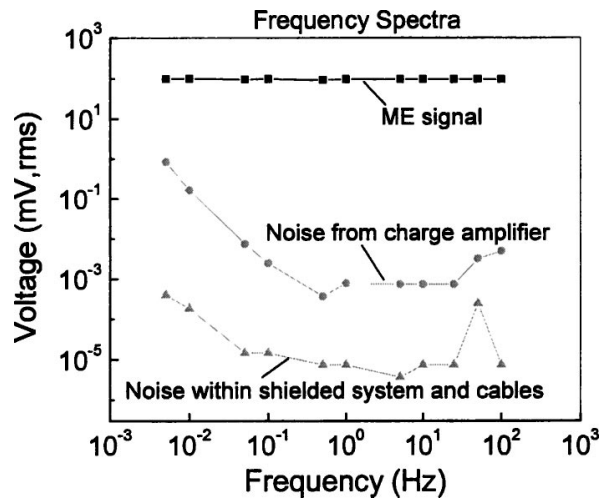

FIG. 2. ME voltage and noise equivalent voltage (NEV) as a function of magnetic field frequency in the range of $f=5 \mathrm{mHz}-100 \mathrm{~Hz}$ for 11-layer laminate designs. Measurements were performed using constant ac magnetic fields of $H_{\mathrm{ac}}=10^{-4} \mathrm{~T}$ and dc magnetic bias of $H_{\mathrm{dc}}=0.04 \mathrm{~T}$.

induced by a magnetic field, which could then either (i) be displayed on an oscilloscope-i.e., a time domain measurement without averaging; or (ii) be used in conjunction with a lock-in amplifier, which could further reject noise. We will report results that demonstrate the ability to detect minute magnetic signal variations of low frequencies by method (i), and frequency spectra determined using a swept-sine mode by (ii). All results to be shown were taken under a dc magnetic bias of $H_{\mathrm{dc}}=0.04 \mathrm{~T}$.

Figure 2 shows the induced ME voltage of our ME-MLC as a function of frequency (swept-sine mode) of the magnetic field for $10^{-3}<f<10^{3} \mathrm{~Hz}$. These data were taken under a constant ac magnetic field of $H_{\mathrm{ac}}=10^{-4} \mathrm{~T}$. In this figure, it can clearly be seen that our ME-MLC has a very flat frequency response: no significant roll of the induced voltage was observed with decreasing measurement frequency over the investigated bandwidth from an extremely low frequency of $\sim 5 \times 10^{-3} \mathrm{~Hz}$ to those in excess of $10^{2} \mathrm{~Hz}$. The results clearly demonstrate (i) the lack of an $f_{\text {cut }}$ for the ME-MLC, at least until the milli-Hz range; and accordingly (ii) that our ME-MLCs have the potential to function as a low frequency (quasistatic) and compact magnetic field sensor. Clearly, a higher $R$ and a ME laminate with more piezoelectric layers will have a longer $\tau$, or a lower $f_{\text {cut }}$, thus, a "quasistatic" magnetic field can be detected.

Next, to illustrate the potential for detection of small magnetic field variations at low frequencies, we determined the equivalent noise floor in our magnetically shielded environment. This was done by setting the ac magnetic drive to $H_{\mathrm{ac}}=0$ and the dc magnetic bias to $H_{\mathrm{dc}}=0.04 \mathrm{~T}$, and subsequently measuring the noise-equivalent voltage (NEV) of the sample as a function of frequency. Without using a charge amplifier, we found the NEV of the specimen in the shielded environment to be quite small. In the frequency range of $10^{-1}-10^{1} \mathrm{~Hz}$, the NEV was about $10^{-6} \mathrm{mV}$, which is about a factor of $\sim 10^{-7}$ less than that of the corresponding ME signal in Fig. 2; whereas in the range of $5 \times 10^{-3}-10^{-1} \mathrm{~Hz}$, it was about $10^{-5}-10^{-4} \mathrm{mV}$, or $\sim 10^{-6}-10^{-5}$ of the signal. Upon inserting a charge amplifier, the noise floor was increased in the low frequency range, but remained a factor of $10^{-2}-10^{-5} \times$ smaller than that of the ME signal, as can be seen in Fig. 2. For example, at $f=10^{-2} \mathrm{~Hz}$, the NEV was $\approx 10^{-4} \mathrm{mV}$ without the amplifier, and $10^{-1} \mathrm{mV}$ with it. With respect to the data in Fig. 2 taken under $H_{\mathrm{ac}}=10^{-4} \mathrm{~T}$, this

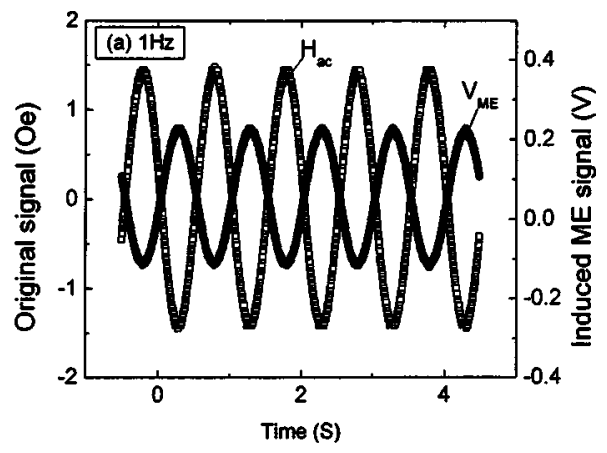

(a)

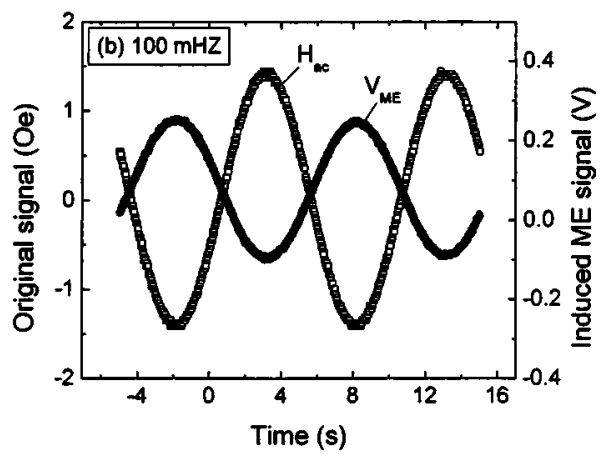

(b)

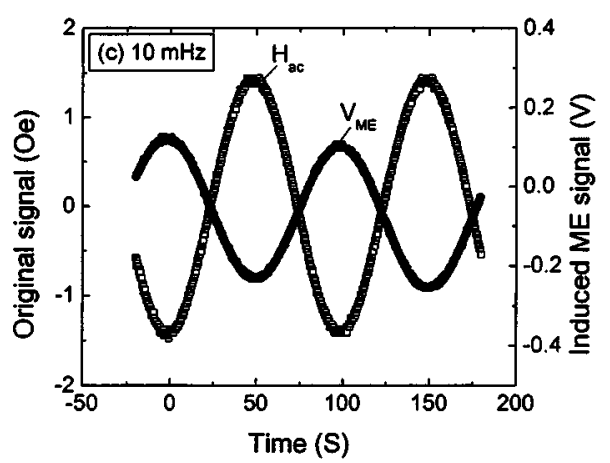

(c)

FIG. 3. Time domain response of the ME sensor to sinusoidal magnetic fields of $H_{\mathrm{ac}}=10^{-4} \mathrm{~T}$ (a) $f=1 \mathrm{~Hz}$; (b) $f=0.1 \mathrm{~Hz}$; and (c) $f=0.01 \mathrm{~Hz}$. The dc magnetic bias of $H_{\mathrm{dc}}=0.04 \mathrm{~T}$.

translates into a sensitivity limit of $\approx 10^{-9}-10^{-7} \mathrm{~T}$ at $\sim 10^{-2} \mathrm{~Hz}$.

Next, we performed studies to demonstrate the ability of the ME-MLC to operate in a time-domain capture mode. Figure 3 shows the ME voltage induced by an ac magnetic field (1 Oe, rms) as a function of time for various frequencies of (a) $1 \mathrm{~Hz}$; (b) $0.1 \mathrm{~Hz}$; and (c) $0.01 \mathrm{~Hz}$. These low frequency results were taken by directly inputting the signal into the oscilloscope, without the use of averaging or phase locking. The induced ME voltage signals were about $110 \mathrm{mV}$ rms that exactly followed the magnetic signal changes. The results demonstrate the ability of our ME-MLC to detect low frequency magnetic fields in a time-domain capture mode, similar to that which will be required in the operational field as a deployable magnetic anomaly detector.

Finally, we illustrate that the ME-MLC sensor can detect much more minute magnetic fields at low frequencies. Figure 4 shows the induced $\mathrm{ME}$ voltage as a function of $H_{\mathrm{ac}}$ for 


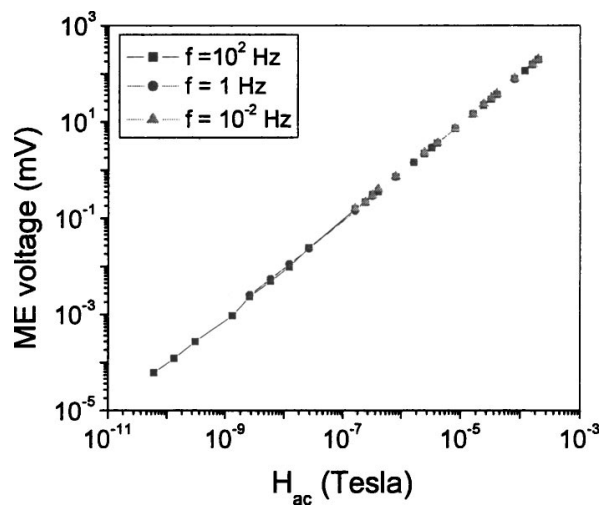

FIG. 4. Demonstration of ability of MLC-ME sensor to detect low frequency minute magnetic field variations at frequencies of $f=10^{-2}, 1$, and $10^{2} \mathrm{~Hz}$. The dc magnetic bias of $H_{\mathrm{dc}}=0.04 \mathrm{~T}$.

frequencies of $10^{-2}, 1$, and $10^{2} \mathrm{~Hz}$. Inspection of this figure will reveal the ability to detect minute field variations on the order of $10^{-7}, 10^{-9}$, and $10^{-11} \mathrm{~T}$ for frequencies of $f=10^{-2}$, 1 , and $10^{2} \mathrm{~Hz}$, respectively. Assuming that the signal-tonoise $(\mathrm{S} / \mathrm{N})$ ratio can be lowered to that shown in Fig. 2, it should be possible to achieve sensitivities of $10^{-9}-10^{-10} \mathrm{~T}$ and below in the milli-Hz frequency range. Preliminary investigations have shown that the S/N ratio of our ME-MLC in practice is limited by pyroelectric and static-electric background noise, rather than either by the noise from the charge amplifier or from the shielded system and cables. Accordingly, we are currently working on gradiometric-like laminate configurations that help auto compensate these noise contributions, which in return will significantly enhance the $\mathrm{S} / \mathrm{N}$ at extremely low frequencies.

Due to low frequency capabilities, our ME-MLC may offer applications in magnetic anomaly detection. ME-MLCs could occupy a unique niche in magnetic sensor technologies, as there is no other simple and/or cost-effective alternative available for detection of small magnetic fields of low frequency that can operate in a passive mode enabling longterm deployment. Our findings open this possibility of - and with future enabling improvements in $\mathrm{S} / \mathrm{N}$-developing a small passive magnetic anomaly detector with pico-T resolution in the frequency domain of $10^{-3}<f<10^{3} \mathrm{~Hz}$.

In summary, we have significantly lowered the limit of the working frequency of a MLC ME laminate by increasing $\tau$. Our results demonstrate that small magnetic field variations of milli-Hz frequencies can be detected by ME-MLCs in a time-domain capture mode. This opens up the possibility of developing ME-MLC magnetic anomaly detectors in the near future.

The authors gratefully acknowledge the support of the Office of Naval Research under Grant Nos. N000140210340, N000140210126, and MURI N000140110761.

${ }^{1}$ L. D. Landau and E. Lifshitz, Electrodynamics of Continuous Media (Pergamon, Oxford, 1960), p. 119.

${ }^{2}$ M. Avellaneda and G. Harshe, J. Intell. Mater. Syst. Struct. 5, 501 (1994).

${ }^{3}$ T. Wu and J. Huang, Int. J. Solids Struct. 37, 2981 (2000).

${ }^{4}$ K. Mori and M. Wuttig, Appl. Phys. Lett. 81, 100 (2002).

${ }^{5}$ J. Ryu, A. V. Carazo, K. Uchino, and H. Kim, Jpn. J. Appl. Phys., Part 1 40, 4948 (2001)

${ }^{6}$ J. Ryu, S. Priya, K. Uchino, H. Kim, and D. Viehland, J. Korean Ceram. Soc. 39, 813 (2002)

${ }^{7}$ C. W. Nan, L. Liu, N. Cai, J. Zhai, Y. Ye, and Y. H. Lin, Appl. Phys. Lett. 81, 3831 (2002).

${ }^{8}$ C.-W. Nan, G. Liu, and Y. Lin, Appl. Phys. Lett. 83, 21 (2003)

${ }^{9}$ G. Srinivasan, E. Rasmussen, B. Levin, and R. Hayes, Phys. Rev. B 65 , 134402 (2002).

${ }^{10} \mathrm{Y}$. Li, vol. 1 (sensors online: http:/sensorsmag.com/articles/1000/52/ index.htm)

${ }^{11}$ S. Dong, F. Bai, J. F. Li, and D. Viehland, IEEE Trans. Ultrason. Ferroelectr. Freq. Control 501236 (2003).

${ }^{12}$ S. Dong, J. F. Li, and D. Viehland, Appl. Phys. Lett. 83, 2265 (2003).

${ }^{13}$ T. Clem, Nav. Res. Rev. 3, 29 (1997).

${ }^{14}$ J. Vrba, SQUID Sensors: Fundamentals, Fabrication and Applications, edited by H. Weinstock (Kluwer, Dordrecht, 1996), p. 117.

${ }^{15} \mathrm{~J}$. Vrba and S. Robinson, Methods 25, 249 (2001).

${ }^{16}$ J. Podney, US Patent No. 5,675,252. 